\title{
Inertial waves and modes excited by the libration of a rotating cube
}

\author{
Jean Boisson, ${ }^{1, a)}$ Cyril Lamriben, ${ }^{1, b)}$ Leo R. M. Maas, ${ }^{2, c)}$ \\ Pierre-Philippe Cortet, ${ }^{1, \mathrm{~d})}$ and Frédéric Moisy ${ }^{1, \mathrm{e})}$ \\ ${ }^{1}$ Laboratoire FAST, CNRS, Université Paris-Sud, UPMC, France \\ ${ }^{2}$ NIOZ Royal Netherlands Institute for Sea Research, Texel, The Netherlands
}

(Received 8 March 2012; accepted 9 June 2012; published online 9 July 2012)

\begin{abstract}
We report experimental measurements of the flow in a cubic container submitted to a longitudinal libration, i.e., a rotation modulated in time. Velocity fields in a vertical and a horizontal plane are measured in the librating frame using a corotating particle image velocimetry system. When the libration frequency $\sigma_{0}$ is smaller than twice the mean rotation rate, $\Omega_{0}$, inertial waves can propagate in the interior of the fluid. At arbitrary excitation frequencies $\sigma_{0}<2 \Omega_{0}$, the oscillating flow shows two contributions: (i) a basic flow induced by the libration motion, and (ii) inertial wave beams propagating obliquely upward and downward from the horizontal edges of the cube. In addition to these two contributions, inertial modes may also be excited at some specific resonant frequencies. We characterize in particular the resonance of the mode of lowest order compatible with the symmetries of the forcing, noted $[2,1,+]$. By comparing the measured flow fields to the expected inviscid inertial modes computed numerically [L. R. M. Maas, "On the amphidromic structure of inertial waves in rectangular parallelepiped," Fluid Dyn. Res. 33, 373 (2003)], we show that only a subset of inertial modes, matching the symmetries of the forcing, can be excited by the libration. ( 2012 American Institute of Physics. [http://dx.doi.org/10.1063/1.4731802]
\end{abstract}

\section{INTRODUCTION}

Rotating fluids support the existence of a singular class of waves called inertial waves, ${ }^{1-3}$ which are anisotropic and propagate because of the restoring nature of the Coriolis force. These waves exist only for excitation frequencies $\sigma_{0}$ lower than twice the rotation rate $\Omega_{0}$. In a confined volume of fluid, inertial waves may become phase-coherent and experience a resonance due to multiple reflections over the container walls, leading to the so-called inertial modes. These inertial modes are relevant to geophysical and astrophysical flows (in liquid cores of planets and stars), but also to mechanical engineering flows (e.g., in liquid-filled projectiles).

Inviscid inertial modes are the eigenmodes of a given container geometry, and can be found in general when the walls are either normal, or parallel to the rotation axis, ${ }^{1,4}$ but also in some specific cases such as spheres, spheroids ${ }^{5}$ and, to some extent, in spherical shells (namely for so-called R-modes that lack radial displacement). However, in general, when sloping walls are present, the wave focusing and defocusing induced by the peculiar reflection law of inertial waves ${ }^{6}$ precludes the existence of inviscid eigenmodes, and the concentration of energy along particular beams leads to wave attractors. ${ }^{7}$ The resonance frequencies of inviscid inertial modes can be derived analytically only in some specific geometries, such as cylinders, spheres, and spheroids. ${ }^{1,5,8}$ In the case of a

\footnotetext{
a)Electronic mail: boisson@fast.u-psud.fr.

b) Electronic mail: lamriben@ @ast.u-psud.fr.

c) Electronic mail: Leo.Maas@nioz.nl.

d)Electronic mail: ppcortet@fast.u-psud.fr.

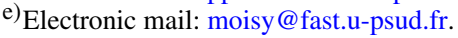


parallelepipedic container, such as used in the experiments presented here, the frequencies and the spatial structure of the inviscid inertial modes have been determined numerically. ${ }^{9}$

In geophysical and astrophysical flows, several types of forcing may be at the origin of the excitation of inertial modes. Inertial modes have been excited experimentally in a spherical cavity by a longitudinal libration ${ }^{10}$-i.e., a time modulation of the rotation rate-and have recently been described in numerical simulations in spheres and spherical shells. ${ }^{1-14}$ Precession ${ }^{15-17}$ and periodic deformation of the walls modeling gravitational tides ${ }^{18,19}$ are other examples of forcing, providing an efficient generation of inertial modes. These inertial modes have been proposed to contribute, through nonlinear self-interaction, to the generation of steady zonal flows, which are visible for instance in the atmosphere of gaseous planets like Jupiter. ${ }^{19-21}$

Although axisymmetric geometries have been primarily considered to observe and characterize inertial modes, non-axisymmetric geometries such as parallelepipeds ${ }^{9}$ are also of fundamental interest. In particular, the symmetries of the container, the symmetries of the forcing, and the role of viscosity, are critical parameters in determining which inertial modes can be excited.

Inertial modes may be present in any laboratory experiment performed in a rotating container. They have been for instance detected in ensemble averages of turbulence generated by the translation of a grid in a rotating container. ${ }^{22-24}$ When present, these modes may couple to the turbulence, and have a profound influence on its statistical properties. In particular, turbulence in the presence of inertial modes, which are non homogeneous by nature, cannot be considered as freely decaying, raising the issue of the relevance of the homogeneous framework to describe rotating turbulence in laboratory experiments.

In this paper, we investigate the spatial structure of the oscillating flow generated by the longitudinal libration of a cube, and we characterize the efficiency of this configuration to excite inertial modes. Measurements are performed both in a vertical and in a horizontal plane (the rotation axis is vertical) using a co-rotating two-dimensional particle image velocimetry (PIV) system. For libration frequencies lower than twice the rotation rate $\sigma_{0}<2 \Omega_{0}$, we observe, in addition to the basic oscillating flow induced by the libration, the propagation of oblique inertial wave beams emitted from the horizontal edges of the cube. These wave beams, similar to the ones observed in cylindrical geometry, ${ }^{4,25}$ originate from the convergence of Ekman fluxes near the edges of the container. In addition, for a specific libration frequency in the explored range $\sigma_{0} / 2 \Omega_{0} \in[0.60 ; 0.73]$, libration also excites an inertial mode, of spatial structure in close agreement with the inviscid computation of Ref. 9. Our results suggest that, among all the eigen modes predicted numerically, only a small subset of low order modes matching exactly the symmetries of the cube libration can be actually excited.

\section{EXPERIMENTAL SETUP}

\section{A. Flow geometry and rotating platform}

The experimental setup, sketched in Fig. 1, consists in a closed cubic glass tank, of inner size $2 L=30 \mathrm{~cm}$, filled with water and mounted on a precision rotating turntable of $2 \mathrm{~m}$ in diameter. We use a coordinate system centered on the horizontal square, $-L \leq x, y \leq L$, with the horizontal walls at $z=0$ and $z=2 L$, perpendicular to the rotation vector $\boldsymbol{\Omega}=\Omega(t) \mathbf{e}_{z}$. The mean angular velocity of the rotating platform is set to $\Omega_{0}=0.419 \mathrm{rad} \mathrm{s}^{-1}(4 \mathrm{rpm})$. The Ekman number of the system, which compares the viscous to the Coriolis force, is $E=v /\left(2 \Omega_{0} L^{2}\right)=5.3 \times 10^{-5}$. The rotation of the fluid is set long before the start of the libration, at least $1 \mathrm{~h}$, in order for transient spin-up recirculations to be damped. Once the solid-body rotation is reached, the turntable is submitted to a longitudinal libration, which consists in a modulation of the angular velocity $\Omega(t)$ around $\Omega_{0}$ at a frequency $\sigma_{0}$ with a peak-to-peak amplitude $2 \epsilon \Omega_{0}$,

$$
\Omega(t)=\Omega_{0}\left[1+\epsilon \cos \left(\sigma_{0} t\right)\right] .
$$

In the frame rotating at constant velocity $\Omega_{0}$, the libration of the cube is described by the angle

$$
\varphi(t)=\epsilon \frac{\Omega_{0}}{\sigma_{0}} \sin \left(\sigma_{0} t\right)
$$




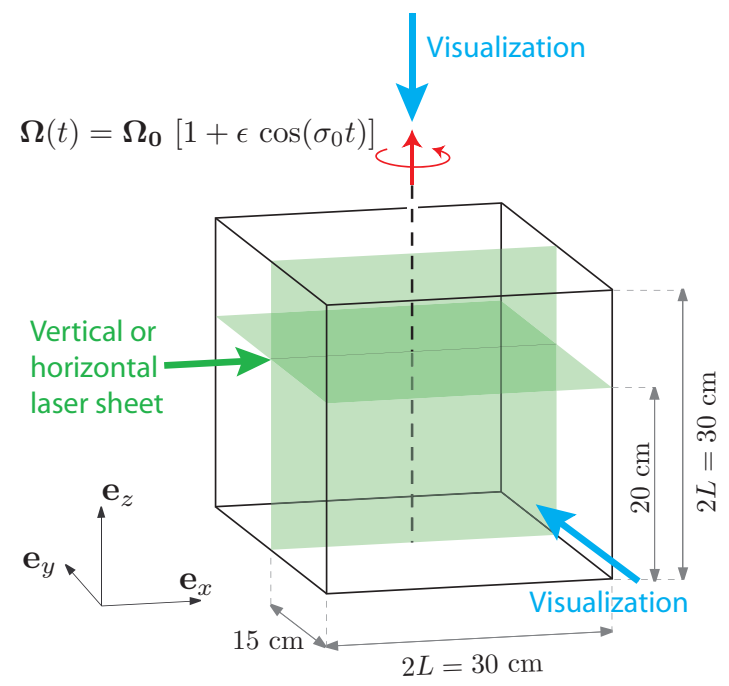

FIG. 1. Schematic view of the $(30 \mathrm{~cm})^{3}$ water tank mounted on the rotating platform. The bottom and top walls are located at $z=0$ and $z=2 L$, and the origin of the coordinates $(x, y)$ is taken at the center of the square cross section. Two-dimensional PIV measurements are achieved in the vertical plane $y=0$ and in the horizontal plane $z=4 L / 3$ in the librated frame using a corotating laser sheet and a camera aiming normally at it.

The normalized libration amplitude $\epsilon$, which is the Rossby number of the problem, is varied between $2 \%$ and $16 \%$. In order to excite inertial waves, the libration frequency $\sigma_{0}$ can take in principle any value in the range $\left[0,2 \Omega_{0}\right]$. In this paper, we focus on the restricted range $\sigma_{0} / 2 \Omega_{0}$ $\in[0.60 ; 0.73]$. For these frequencies, the peak-to-peak libration angle $\Delta \varphi=2 \epsilon \Omega_{0} / \sigma_{0}$ lies in the range from $1^{\circ}$ to $13^{\circ}$ for $\epsilon=2 \%$ to $16 \%$. The relative precision in the control of the instantaneous rotation rate is better than $10^{-3}$. After the start of the libration, we wait at least half an hour before the data acquisition (which represents $8 \tau_{E}$, where $\tau_{E}=L\left(\nu \Omega_{0}\right)^{-1 / 2}$ is the Ekman time scale) in order to reach a stationary regime.

\section{B. Particle image velocimetry (PIV) measurements}

Velocity fields are measured in the librated reference frame using a two-dimensional PIV system mounted on the rotating platform (Fig. 1). ${ }^{26,27}$ Measurements are performed either in the vertical plane $y=0$ or in the horizontal plane $z=4 L / 3=20 \mathrm{~cm}$. This last particular choice is motivated by the fact that $z=4 L / 3$ does not correspond to a node for the inertial modes considered in this paper (the centered horizontal plane $z=L$ is a node for the inertial modes of even vertical wavenumber $n$ ). The flow is seeded with $10 \mu \mathrm{m}$ tracer particles, and illuminated by a corotating laser sheet generated by a $140 \mathrm{~mJ}$ Nd:YAG pulsed laser. For both horizontal and vertical measurements, the entire $30 \times 30 \mathrm{~cm}^{2}$ flow sections are imaged through the transparent sides of the tank with a high resolution $2048 \times 2048$ pixels camera aiming normally at the laser sheet.

Each acquisition consists in at least 1000 , and up to 3000 , images taken at a sampling rate between $12 \sigma_{0}$ and $36 \sigma_{0}$, which correspond to at least 80 libration periods. The sampling rate is chosen according to the libration amplitude in order to keep a typical particle displacement of the order of 5 pixels between two successive images. PIV fields are computed over successive images using $32 \times 32$ pixels interrogation windows with $50 \%$ overlap, leading to a spatial resolution of $3.5 \mathrm{~mm}$. This resolution is not enough to resolve the thickness of the Ekman boundary layers, $\delta_{E}=L E^{1 / 2} \simeq 1 \mathrm{~mm}$, but is appropriate for the flow structures associated to inertial waves and modes in the bulk of the fluid. 


\section{BASIC LIBRATION FLOW}

\section{A. Inviscid solution}

We first characterize the basic flow response to the libration forcing. Since in the present experiments the modulation period $2 \pi / \sigma_{0} \simeq[20 ; 25] \mathrm{s}$ is much shorter than the Ekman time $\tau_{E}$ $\simeq 230 \mathrm{~s}$ (which is the time scale required for the angular velocity of the fluid to match that of the boundaries), the core of the flow can be considered as essentially inertial, and rotating at the constant rotation rate $\Omega_{0}$. In the frame rotating at $\Omega_{0}$, the flow is therefore approximately at rest, and surrounded by oscillating recirculations induced by the periodic motion of the walls.

The inviscid response to the libration of an arbitrary container, whose walls are either parallel, or normal to the rotation axis, can be derived by assuming that the flow is strictly two-dimensional. In the absence of viscosity, the absolute vorticity of the fluid in the frame of the laboratory,

$$
\omega_{a}=\omega+2 \Omega(t) \mathbf{e}_{z},
$$

must be conserved, and hence given by the mean vorticity $2 \Omega_{0} \mathbf{e}_{z}$. Here, $\omega$ is the relative vorticity, as measured in the libration frame. If viscosity is present, this result remains approximately valid far from the boundaries, with the additional assumption that $\sigma_{0} / \Omega_{0} \gg E^{1 / 2}$ (i.e., for a rapid libration compared to the Ekman time scale). With the total angular velocity of the libration given by Eq. (1), the relative vorticity $\omega$ is therefore vertical, homogeneous, and given by

$$
\omega_{z}(t)=\frac{\partial u_{y}}{\partial x}-\frac{\partial u_{x}}{\partial y}=-2 \epsilon \Omega_{0} \cos \left(\sigma_{0} t\right) .
$$

In an axisymmetric container, the resulting flow is an oscillating solid body rotation of angular velocity $-\epsilon \Omega_{0} \cos \left(\sigma_{0} t\right)$ : this simply describes a fluid at rest in the frame rotating at constant rate $\Omega_{0}$. The case of a non-axisymmetric container is more complex, and can be solved in terms of a streamfunction $\psi(x, y, t)=\Psi(x, y) \cos \left(\sigma_{0} t\right)$, such that $\left(u_{x}, u_{y}\right)=\left(-\partial_{y} \psi, \partial_{x} \psi\right)$. Equation (3) therefore takes the form of a Poisson equation for the spatial part of the streamfunction,

$$
\Delta \Psi=-2 \epsilon \Omega_{0} .
$$

The solution of this equation for a square domain, subject to the condition that the streamfunction vanishes at the boundary, is given in the Appendix, in Eq. (A3). The streamlines are circular near the center of the container, as in a solid-body rotation, but they become gradually more square near the boundaries (see the velocity field in Fig. 2(a)).

(a) Inviscid theory

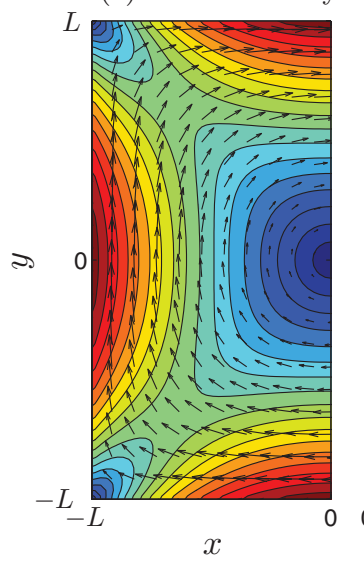

(b) Experiment

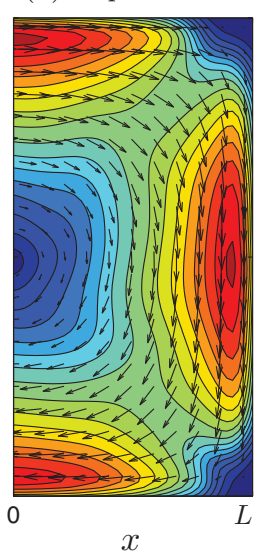

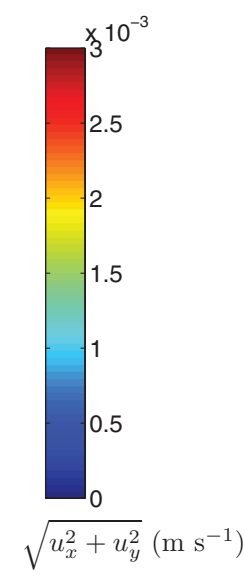

FIG. 2. Velocity fields excited by libration in a container of square cross-section. (a) Inviscid solution, computed from Eq. (A3). (b) PIV measurements at the phase $\sigma_{0} t=0$ (maximum libration velocity). The libration frequency is $\sigma_{0} / 2 \Omega_{0}$ $=0.648$ (corresponding to the mode $[2,2,+]$, which is not resonant in this experiment), and its amplitude is $\epsilon=0.04$. 


\section{B. Experimental measurements of the libration flow}

Figure 2 compares the measured libration flow obtained by PIV and the inviscid solution (A3). The velocity field shown here is taken at the phase $\sigma_{0} t=0$ corresponding to the maximum amplitude of the libration, when the flow in the librated frame is anticyclonic (i.e., $\omega_{z}=-2 \epsilon \Omega_{0}$ ). The libration frequency $\sigma_{0}$ is chosen here far from any resonant frequency of inertial mode. The agreement between the measured field and the inviscid solution is excellent in most of the flow section, except near the boundaries. It must be noted that the vanishing of the velocity near the boundaries may be affected by the PIV resolution (about $3 \mathrm{~mm}$ ), which is of the same order as the expected boundary layer thickness.

An interesting feature of Fig. 2(b) is the wavy shape of the experimental iso-velocity lines: this is a first indication that, in addition to the basic libration flow, the flow also contains a wave component. This additional component is further described in Sec. V.

For all the libration amplitudes investigated here ( $\epsilon=2 \%$ to $16 \%)$, the fluid response to the forcing is found to remain essentially linear. This is demonstrated in Fig. 3, where the temporal energy spectra $E(\sigma)$ are shown for different forcing amplitude $\epsilon$. Here, the spectrum is defined as $E(\sigma)=\left\langle\left|\hat{\mathbf{u}}_{\sigma}\right|^{2}\right\rangle_{x y}$, where $\hat{\mathbf{u}}_{\sigma}(x, y)$ is the temporal Fourier transform computed at each location, and \langle\rangle$_{x y}$ is the spatial average over the horizontal plane. The Fourier transform is computed over at least 80 periods of libration.

All spectra show a narrow peak at the forcing frequency $\sigma_{0}$, well above the white noise level of the PIV measurements. We note that, since we are only interested here in forcing frequencies $\sigma_{0}>\Omega_{0}$, higher harmonics $n \sigma_{0}$, if present, are beyond the upper limit $2 \Omega_{0}$, and are therefore not governed by the dynamics of inertial waves.

We can note that, for all forcing amplitudes $\epsilon$, the peak at $\sigma_{0}$ contains at least $97 \%$ of the total flow energy. The remaining energy is associated to secondary peaks at $\sigma=0, \sigma=\Omega_{0}$, and higher harmonics $\left(\sigma>2 \Omega_{0}\right)$. The secondary peak at $\sigma=\Omega_{0}$, mostly visible at low $\epsilon$, corresponds to a residual fluid motion synchronized with the platform rotation rate. The peak at $\sigma=0$ can be associated to the generation of a weak mean flow. This mean flow is probably a nonlinear response to the libration forcing, and is not investigated in the present paper.

In order to improve the signal-to-noise ratio, we perform, in the following, a band-pass filtering of the velocity fields at the forcing frequency $\sigma_{0}$. This procedure consists in filtering the Fourier transform $\hat{\mathbf{u}}_{\sigma}$ at all $\sigma$ except in a narrow region centered on $\sigma_{0}$ of width $\delta \sigma / 2 \Omega_{0}=0.024$ (which

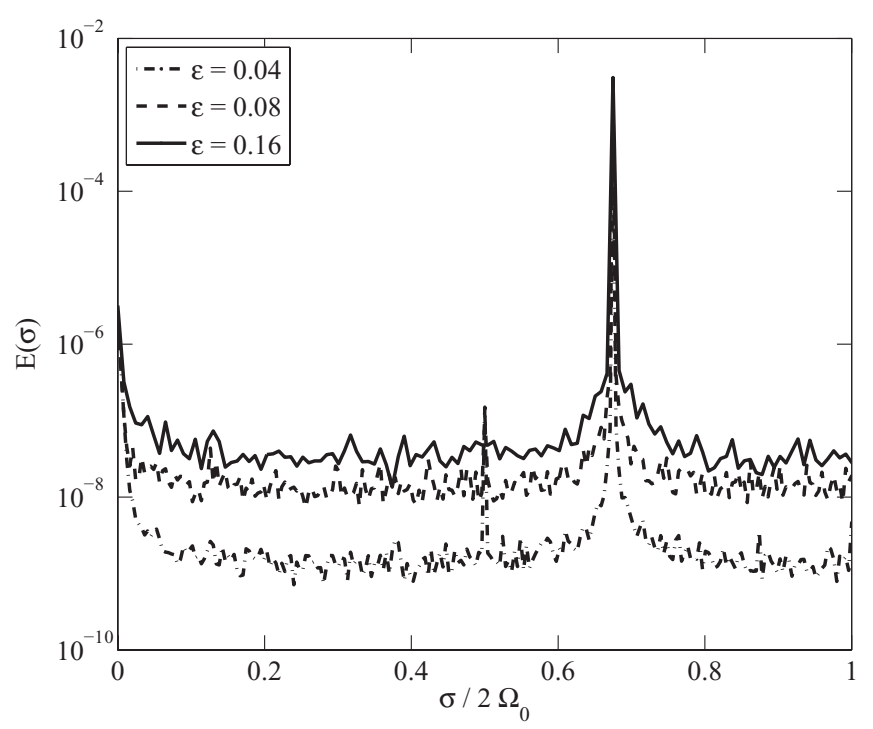

FIG. 3. Temporal energy spectra $E(\sigma)$ for a forcing frequency $\sigma_{0} / 2 \Omega_{0}=0.675$ (corresponding to the mode $[2,1,+]$ ), for three libration amplitudes $\epsilon=0.04,0.08$, and 0.16 . 
contains the energy of the peak to within 1\%), and computing the inverse Fourier transform of the resulting filtered field.

\section{INVISCID INERTIAL MODES AND SYMMETRIES OF THE LIBRATION FORCING}

\section{A. Inviscid inertial modes}

The eigen frequencies and the spatial structures of the inertial modes in a parallelepiped whose sides are parallel or perpendicular to the rotation axis have been determined numerically for an inviscid fluid in Ref. 9. These predictions have been achieved from the numerical resolution of the eigenproblem defined from the inviscid linearized equations in a rotating frame. Any frequency $\sigma_{0}$ in the range $\left[0,2 \Omega_{0}\right]$ corresponds to an inertial mode. Accordingly, an infinity of inviscid modes may be excited if the system is forced at a given frequency with a finite bandwidth.

Following the notation introduced in Ref. 24, we label the inertial modes as $[n, m, s]$. The first index $n$ is the normalized vertical wavenumber, such that the velocity field $\mathbf{u}$ shows $n$ recirculation cells in the vertical direction. With the container walls taken at $z=0$ and $z=H$, the velocity field has the form

$$
\begin{aligned}
\mathbf{u}_{\perp}(x, y, z, t) & =\cos \left(\pi n \frac{z}{H}\right) \breve{\mathbf{u}}_{\perp}(x, y, t), \\
u_{z}(x, y, z, t) & =\sin \left(\pi n \frac{z}{H}\right) \breve{u}_{z}(x, y, t),
\end{aligned}
$$

with $\mathbf{u}_{\perp}=u_{x} \mathbf{e}_{x}+u_{y} \mathbf{e}_{y}$ (we restrict to $H=2 L$ in this paper). Inertial modes are therefore stationary in the vertical direction, but their horizontal structure $\breve{\mathbf{u}}(x, y, t)$ may be either stationary (the so-called "sloshing" modes), or propagating. The second index $m$ enumerates the eigen frequencies of modes of sign $s$ from the largest one $(m=1)$ down. Larger values of $m$ essentially correspond to finer structures in the horizontal plane. Since each mode $m$ is expressed in terms of an infinite amount of horizontal Fourier modes, the index $m$ is not directly related to a number of nodes as for the vertical index $n$. Finally, the sign $s$ refers to the invariance of the mode with respect to the rotation of angle $\pi$ about the $z$ axis. More precisely, a mode $\mathbf{u}(x, y, z, t)$ has symmetry $s$ if

$$
\left(\begin{array}{l}
u_{x} \\
u_{y} \\
u_{z}
\end{array}\right)(-x,-y, z, t)=s\left(\begin{array}{c}
-u_{x} \\
-u_{y} \\
u_{z}
\end{array}\right)(x, y, z, t) .
$$

The resonance frequencies of inertial modes $[n, m, s]$ are increasing functions of $n$ and, at fixed $n$ and $s$, decreasing functions of $m$. This behavior essentially originates from the dispersion relation for plane inertial waves,

$$
\sigma=2 \Omega_{0} \cos \theta
$$

where $\theta$ is the angle between the wavevector $\mathbf{k}$ and the rotation axis, $\cos \theta=k_{z} /|\mathbf{k}|$. Low frequencies $\sigma$ are therefore associated to nearly horizontal $\mathbf{k}$, i.e., to small $k_{z}$ and hence to small $n$, or/and to large $k_{x, y}$ and hence to large $m$.

\section{B. Symmetry properties and boundary conditions for a viscous fluid}

In the case of a viscous fluid, the no-slip boundary condition at the walls is expected to affect the spatial structure of the inertial modes. The spectrum and spatial structure of viscous inertial modes in a rotating parallelepiped have not been computed yet. It is therefore of first interest to check as to what extent the inviscid modes found numerically could be recovered in a viscous fluid at a finite Ekman number. Since viscosity damps preferentially the high order modes (i.e., the modes with large indices $n$ and/or $m$ ), we expect the libration to force more efficiently modes of low order. This is straightforward for the vertical wavenumber $n$, which is naturally associated to a viscous damping proportional to $v n^{2}$. No equivalent simple law exists for the horizontal index $m$, but we can similarly expect that lower $m$, associated to larger scales in the horizontal plane, will be favored in the presence of viscosity. 
TABLE I. List of all the inviscid inertial modes $[n, m, s]$, truncated at $n \leq 4$ and $m \leq 6$, in the range of frequencies $\sigma_{0} / 2 \Omega_{0} \in[0.60 ; 0.73] . n$ is the normalized vertical wavenumber, $m$ characterizes the horizontal structure, and $s= \pm$ refers to the symmetry or antisymmetry of the mode with respect to rotation of angle $\pi$ about the rotation axis (see Ref. 9 for details). The $*$ symbol marks the four modes having symmetries compatible with the libration forcing: even $n$ and $s=+$.

\begin{tabular}{lr}
\hline \hline $\begin{array}{l}\text { Mode } \\
{[n, m, s]}\end{array}$ & $\begin{array}{r}\text { Frequency } \\
\sigma / 2 \Omega_{0}\end{array}$ \\
\hline$[2,1,+]^{*}$ & 0.6742 \\
{$[2,2,+]^{*}$} & 0.6484 \\
{$[3,2,-]$} & 0.7271 \\
{$[3,3,-]$} & 0.6857 \\
{$[3,4,-]$} & 0.6848 \\
{$[3,3,+]$} & 0.6485 \\
{$[3,4,+]$} & 0.6258 \\
{$[4,5,+]^{*}$} & 0.6960 \\
{$[4,6,+]^{*}$} & 0.6945 \\
{$[4,5,-]$} & 0.6889 \\
{$[4,6,-]$} & 0.6643 \\
\hline \hline
\end{tabular}

From the symmetries of the boundary conditions, it is possible to anticipate which inertial modes are compatible with the libration forcing. In the frame rotating at constant velocity $\Omega_{0}$, the angular oscillation of the top and bottom walls is described by the velocity $\mathbf{u}(x, y, z=0)$ $=\mathbf{u}(x, y, z=2 L)=\epsilon \Omega_{0} \cos \left(\sigma_{0} t\right)\left(-y \mathbf{e}_{x}+x \mathbf{e}_{\mathbf{y}}\right)$ which, according to Eq. (6), has symmetry $s=+$. Moreover, the no-slip boundary conditions impose equal horizontal velocity at the top and bottom walls, which is satisfied only for even vertical wavenumbers $n$ (the basic libration flow described in Sec. III is vertically invariant, and is hence associated to $n=0$ ). Finally, there is the possibility that particular symmetries associated to the second index $m$ may even further reduce the set of modes compatible with the symmetries of the libration forcing. According to these symmetry properties, the longitudinal libration is expected to excite only a subset of the modes $[n, m, s]$ among those having even $n$ and $s=+$.

It must be noted that those symmetry arguments state which modes are forbidden by this forcing, but they do not state which modes, among the allowed ones, are effectively excited by the libration. In other words, we would like to address the question of how the fluid motion induced by the oscillating walls, and in particular close to the edges of the cube, will be transmitted to the inertial modes, and to characterize the efficiency of this transmission.

In the following, we investigate the flow response for libration frequencies in the vicinity of two inertial modes of low order allowed by the symmetries of the forcing: $[2,1,+]$ and $[2,2,+]$. More specifically, we systematically characterize the flow in the range $\sigma_{0} / 2 \Omega_{0} \in[0.60 ; 0.73]$ surrounding these two modes. The list of all modes in this range, truncated at $n \leq 4$ and $m \leq 6$, is given in Table I. Among these modes, only the four ones $[2,1,+],[2,2,+],[4,5,+]$, and $[4,6,+]$ (marked by a $*$ symbol) have the correct symmetries (even $n$ and $s=+$ ) and could be observed in principle. However, we will show in the following that viscous effects and additional symmetry properties actually select only the single mode $[2,1,+]$ in this list.

\section{EDGE BEAMS AND INERTIAL MODES}

\section{A. Flow in the vertical plane: Non-resonant case}

We first describe the flow in the vertical plane $y=0$ in the absence of inertial modes. The velocity $\mathbf{u}_{x, z}$ and the vorticity $\omega_{y}(x, z)$ shown in Fig. 4 for $\epsilon=0.02$ are for forcing frequencies $\sigma_{0} / 2 \Omega_{0}$ $=0.60$ (a) and 0.73 (b), chosen far from any low order inertial mode. Fields are not displayed in lateral strips of $12 \mathrm{~mm}$ width along the walls due to poor quality of the data induced by light 


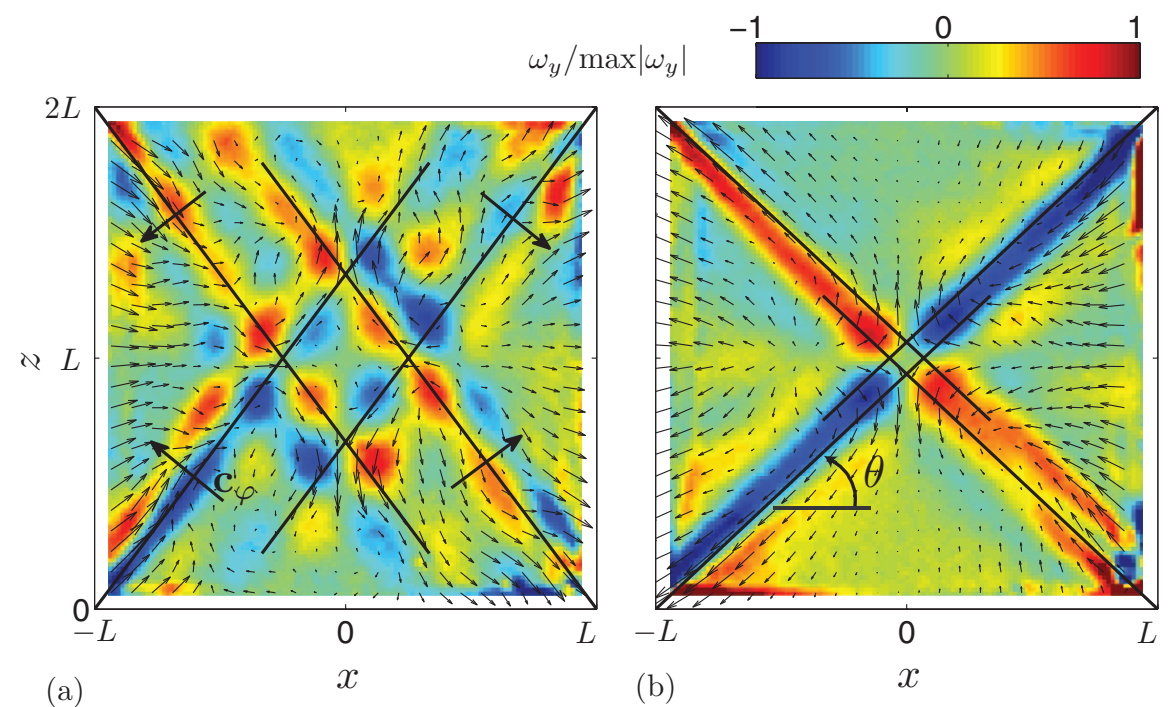

FIG. 4. Spatial structure, in the vertical plane $y=0$, of the oscillating flow excited with $\epsilon=0.02$ and at frequencies $\sigma_{0} / 2 \Omega_{0}$ $=0.60$ (a) and 0.73 (b), in the absence of inertial modes. The velocity fields are extracted by temporal band-pass filtering around the excitation frequency. The arrow fields illustrate the $(x, z)$ components of the velocity field at a given arbitrary phase of the oscillation, and the gray shade/color maps the corresponding normalized vorticity $\omega_{y} / \max \left|\omega_{y}\right|$. Resolution of the velocity fields has been reduced by a factor 5 for better visibility. In (a), bold arrows show the direction of the phase velocity $\mathbf{c}_{\varphi}$ for each wave beam. In (a) and (b), the solid black lines show the expected direction predicted for each wave beam from the dispersion relation, $\cos \theta=\sigma_{0} / 2 \Omega_{0}$.

reflections. Since the basic libration flow is normal to the plane $y=0$, the vertical flows here show the $x$ and $z$ deviations from the libration flow, and contain therefore only the contributions from the wave component of the flow.

We observe that the libration excites a cross shaped pattern made of four oblique shear layers originating from the bottom and top edges of the cube. These shear layers actually correspond to propagative inertial waves in two-dimensional plane wave packets, which contain approximately one wavelength in their transverse direction. There are actually eight two-dimensional wave packets emitted from the four upper and the four lower edges of the container; only the four ones emitted from the edges defined by $x= \pm L, z=0,2 L$ can be seen in the vertical cut $y=0$. The direction of the beams is found in good agreement with the dispersion relation (7), as shown by the black lines in Fig. 4 making an angle $\theta=\cos ^{-1}\left(\sigma_{0} / 2 \Omega_{0}\right)$ with the horizontal. In these wave beams, fluid particles describe an anticyclonic circular motion at frequency $\sigma_{0}$ in the planes inclined at angles $\pm \theta$ with the horizontal (the sign depends on the considered beam). The shearing motion traced by the vorticity $\omega_{y}$ is related to the variation of the wave phase in the direction normal to $\pm \theta$. We also observe that the phase of the wave travels normal to the beam, with a phase velocity $\mathbf{c}_{\varphi}$ directed towards the vertical wall from which originates the beam. In Fig. 4(a) $\left[\theta=\cos ^{-1}(0.60) \simeq 53^{\circ}\right]$, the four wave beams show interferences at their intersections, which make the wave pattern rather complex. On the contrary, in Fig. 4(b) $\left[\theta=\cos ^{-1}(0.73) \simeq 43^{\circ}\right]$, the opposite wave beams are almost aligned for this particular frequency. As a consequence, the wave beams show constructive interferences, leading to almost standing waves along the diagonals, but propagative outwards on their sides.

These edge beams are similar to the ones observed in cylindrical containers, e.g., in the early experiment of McEwan ${ }^{4}$ (forced by a tilted top lid) and in the numerical simulations of Duguet et al. ${ }^{25}$ (forced by an oscillating axial compression). Similar beams are also found in the recent simulations of Sauret et al. ${ }^{28}$ (forced by a longitudinal libration) although in this case, the beam angle is apparently not directly related to the libration frequency, and probably results from the turbulence in the boundary layers over the sidewalls. In all these references, the container is cylindrical, resulting in conical edge beams, emitted from the two circular edges and focusing towards the rotation axis. In our non-axisymmetric geometry, since the disturbance sources are linear segments, there are eight two-dimensional edge beams emitted from the four upper and the four lower edges of the container. 
These edge beams originate from the oscillating outward (resp. inward) Ekman layers on the upper and lower walls associated with the prograde (resp. retrograde) part of the libration motion. Far from the lateral walls, where the streamlines of the libration flow are approximately axisymmetric, this flow is approximately radial, of order $\epsilon \Omega_{0} \sqrt{x^{2}+y^{2}}$. In the classical axisymmetric spin-up problem, the horizontal flow in the unsteady (but slowly varying) Ekman layer is vertically deflected into Stewartson layers along the lateral walls. ${ }^{1}$ However, in the rapidly oscillating situation examined here, the matching condition between the Ekman and Stewartson layers cannot be satisfied, and the flow in the Ekman layer detaches when it reaches the lateral walls, generating an oscillating shear layer in the bulk, ${ }^{16}$ of characteristic velocity $\epsilon \Omega_{0} L$. As a consequence, although the velocity scale of the edge beams is inertial, viscosity is responsible for this flow feature.

The width of these edge beams can be compared to predictions from a viscous theory. Because of viscous damping, it is known that a wave beam spreads as $\ell^{1 / 3}$, where $\ell$ is the distance from the source. ${ }^{29,30}$ More precisely, the thickening of the full-width half maximum $\delta(\ell)$ of a self-similar plane beam emitted by a linear disturbance of small size can be computed, ${ }^{31}$

$$
\delta(\ell) \simeq 6.84 L\left(\frac{E}{\sin \theta}\right)^{1 / 3}\left(\frac{\ell}{L}\right)^{1 / 3},
$$

with $E=v /\left(2 \Omega_{0} L^{2}\right)$. According to this model, a wave beam emitted from an edge reaches the central area of the container after a distance $\ell^{*}=L / \sin \theta$ (for $\theta>45^{\circ}$ ), where its width is $\delta\left(\ell^{*}\right)$ $\simeq 6.84(\sin \theta)^{-2 / 3} L E^{1 / 3} \simeq 4.2-5.0 \mathrm{~cm}$ for the range of angles considered here. This prediction provides correct agreement with the observations in Fig. 4, showing the beams of thickness of order of $3-4 \mathrm{~cm}$ near the center. Note that the case $\theta \simeq 45^{\circ}$ is specific: the overlap of the upper and lower wave beams leads to a different scaling, ${ }^{25} \delta \simeq E^{1 / 4}$.

\section{B. Flow in the vertical plane: Resonant case}

We still examine here the structure of the flow in the vertical plane, but now at frequencies where inertial modes are expected. We select the two frequencies $\sigma_{0} / 2 \Omega_{0}=0.674$ and 0.648 , corresponding to the inviscid predictions for the modes $[2,1,+]$ and $[2,2,+]$, respectively (see Table I). The velocity and vorticity fields are shown in Fig. 5, at the phases $\sigma_{0} t=0(\mathrm{a}, \mathrm{d})$ and $\pi / 2(\mathrm{~b}, \mathrm{e})$ of the libration. In the frame rotating at constant velocity $\Omega_{0}$, the phase $\sigma_{0} t=0$ corresponds to $\varphi=0$ and $\Omega(t)$ $=\epsilon \Omega_{0}$ (i.e., maximum rotation rate), whereas $\sigma_{0} t=\pi / 2$ corresponds to $\varphi$ maximum and $\Omega(t)=0$ [see Eqs. (1)-(2)].

Looking first at the velocity fields (b) and (e), both frequencies show two oscillating cells in the vertical direction, as expected for a mode $n=2$ (see also the movies available at Ref. 32). These oscillating cells are mainly visible at the phase $\sigma_{0} t=\pi / 2$, when the angle $\varphi$ is maximum and the libration motion vanishes. At this phase, the velocity fields clearly satisfy the $s=+$ symmetry, with $u_{x}(-x, z)=-u_{x}(x, z)$ and $u_{z}(-x, z)=u_{z}(x, z)$. On the other hand, at the phase $\sigma_{0} t=0$ corresponding to the maximum libration velocity $(\mathrm{a}, \mathrm{d})$, the $x$ and $z$ components of the velocity associated to this mode $n=2$ vanish and one recovers the propagative edge beams already evidenced in Fig. 4.

Looking now at the vorticity field reveals finer structures of the flow. At phase $\sigma_{0} t=0(\mathrm{a}, \mathrm{d})$, the typical cross shaped pattern composed of four edge beams is found for both forcing frequencies. On the other hand, at phase $\pi / 2(\mathrm{~b}, \mathrm{e})$, the shape of the vorticity field is found to depend now on the forcing frequency. Whereas it keeps approximately its cross-shape structure for $\sigma_{0} / 2 \Omega_{0}=0.648$ (e), it shows four nearly circular extrema of alternate signs for $\sigma_{0} / 2 \Omega_{0}=0.674$ (b). This indicates that, at $\sigma_{0} t=\pi / 2$, the four recirculation cells dominate the vorticity for $\sigma_{0} / 2 \Omega_{0}=0.674$ (b), whereas their vorticity is partially hidden by the strong edge beams for $\sigma_{0} / 2 \Omega_{0}=0.648$ (e).

In order to understand the different behaviors between the two frequencies, we compare now the experimental fields with the numerical predictions of the inviscid modes $[2,1,+]$ and $[2,2,+]$, shown in Figs. 5(c) and 5(f), respectively (movies of the numerical modes are also provided at Ref. 32). Note that the phase of the numerical fields is arbitrary since they are not related to any specific forcing: the numerical fields in Fig. 5 have been simply chosen here at phases which provide 


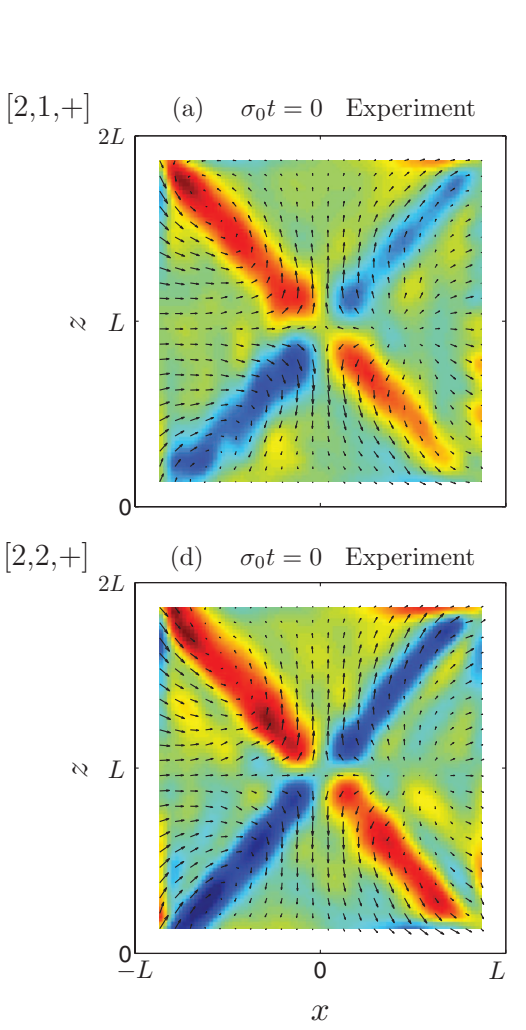

(b) $\sigma_{0} t=\pi / 2 \quad$ Experiment

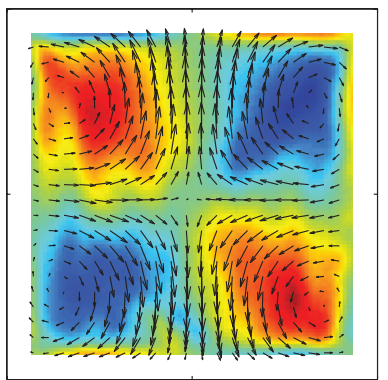

(e) $\quad \sigma_{0} t=\pi / 2 \quad$ Experiment

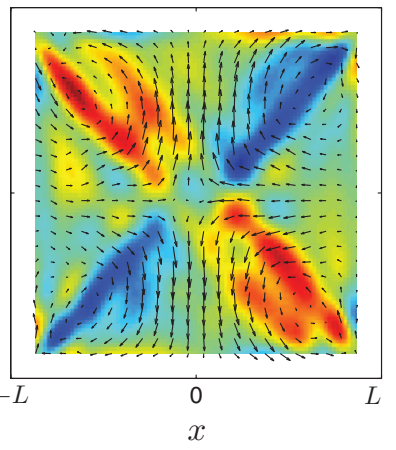

$\omega_{y} / \max \left|\omega_{y}\right|$

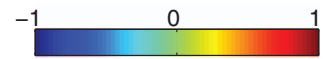

(c) Numerics

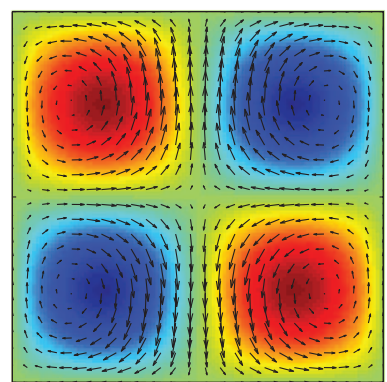

(f) Numerics

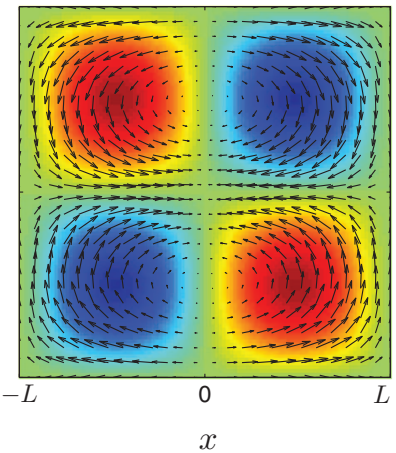

FIG. 5. Spatial structure, in the vertical plane $y=0$, of the oscillating flow excited with $\epsilon=0.04$ and at frequency $\sigma_{0} / 2 \Omega_{0}$ $=0.674$ (a) - (c) and $\sigma_{0} / 2 \Omega_{0}=0.648$ (d) - (f). (a), (b), (d), and (e) correspond to the experimental fields extracted by temporal band-pass filtering around the excitation frequency. (c) and (f) show the corresponding numerical inviscid modes $[2,1,+]$ and $[2,2,+]$ expected at the same frequency. The libration phase is $\sigma_{0} t=0$ for (a) and (d), and $\sigma_{0} t=\pi / 2$ for (b) and (e). The gray shade/color maps the normalized vorticity field $\omega_{y} / \max \left|\omega_{y}\right|$. Resolution of the velocity fields has been reduced by a factor 5 for better visibility (see also Ref. 32).

a good visual correspondence with the experimental fields. Although the vorticity patterns of modes $[2,1,+]$ and $[2,2,+]$ look similar, with four extrema of alternate signs, their velocity fields are very different: the vertical velocity is maximum on the vertical axis for $[2,1,+]$, but it is zero for $[2,2,+]$. The smooth pattern of $\omega_{y}$ found experimentally in Fig. 5(b) matches actually well the prediction for $[2,1,+]$. On the other hand, the comparison fails for Fig. 5(e), both for the velocity and vorticity fields, suggesting that the mode $[2,2,+]$ cannot be excited by the longitudinal libration.

We can interpret these observations as follows. For $\sigma_{0} / 2 \Omega_{0}=0.674$, the flow is a superimposition of the pattern of propagative edge beams and a resonant stationary $[2,1,+]$ mode. Whenever the libration angle is zero (i.e., at phases $\sigma_{0} t=0$ and $\pi$ ), the instantaneous amplitude of the $[2,1,+]$ mode vanishes, and only the edge beams remain visible. Turning now to the case $\sigma_{0} / 2 \Omega_{0}=0.648$, the mode $[2,2,+]$ is apparently not excited, and the edge beam pattern remains visible all the time. Interestingly, it seems that a flow reminiscent of the mode $[2,1,+]$ is excited instead (see Fig. 5(e)), although with a weak amplitude, probably because of the closeness of the eigen frequencies (less than $4 \%$ ) of the two modes. It will indeed be confirmed in Sec. VI that the resonance of the $[2,1,+]$ mode spreads over a significant frequency range because of viscosity.

\section{Flow in the horizontal plane}

We finally turn to visualizations in the horizontal plane $z=4 L / 3$, still for the two frequencies $\sigma_{0} / 2 \Omega_{0}=0.674$ and 0.648 . In order to focus on the wave component of the flow, we remove, in the 

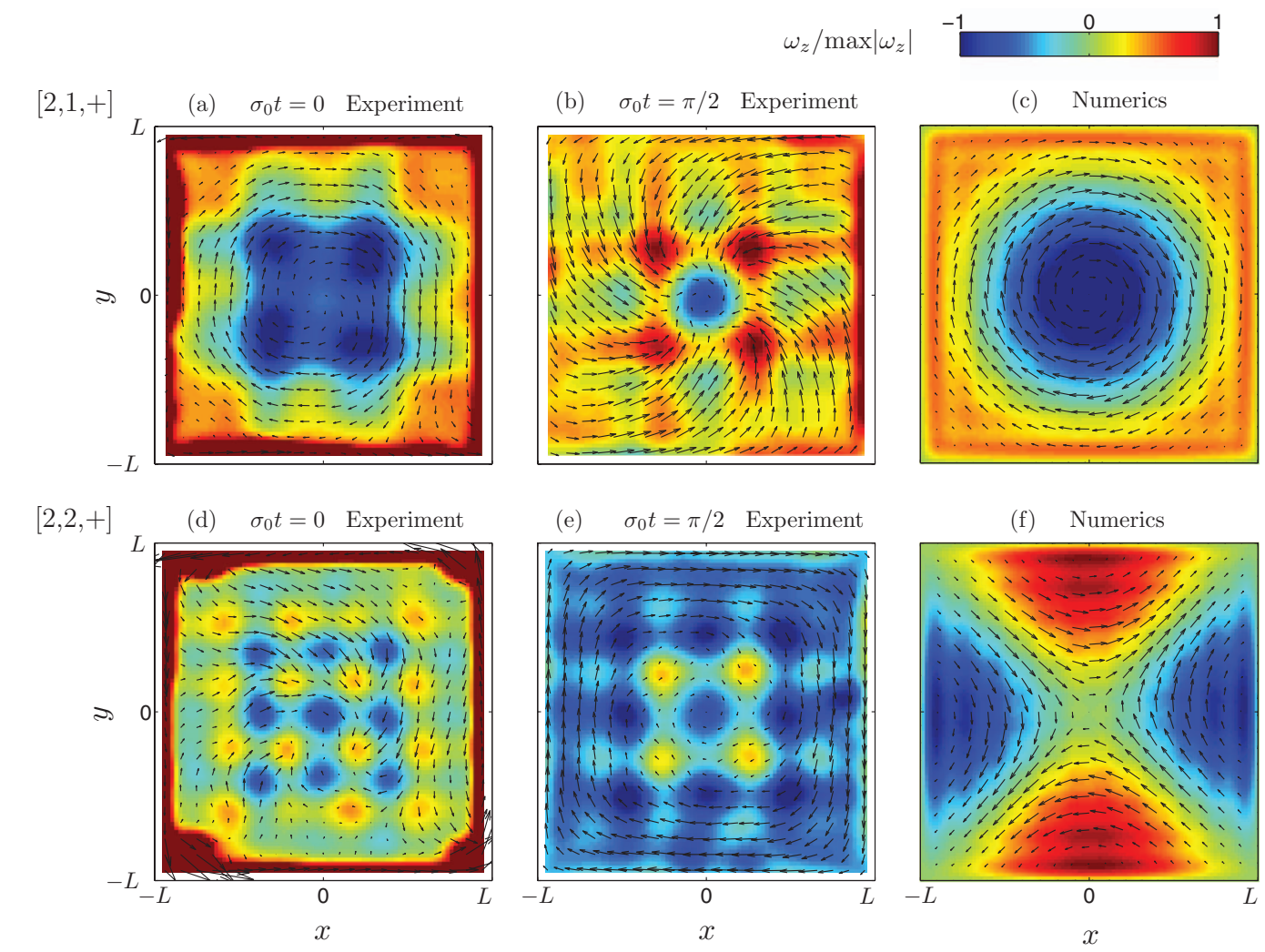

FIG. 6. Spatial structure, in the horizontal plane $z=4 L / 3$, of the wave component of the flow $\mathbf{u}^{\prime}$ excited with $\epsilon=0.04$ and at frequency $\sigma_{0} / 2 \Omega_{0}=0.674$ (a) - (c) and $\sigma_{0} / 2 \Omega_{0}=0.648$ (d) - (f). Same layout as in Fig. 5 .

following, the libration component,

$$
\mathbf{u}^{\prime}(x, y, t)=\mathbf{u}(x, y, t)-\mathbf{u}_{l i b}(x, y) \cos \left(\sigma_{0} t\right),
$$

where $\mathbf{u}(x, y, t)$ is the total velocity measured by PIV, and $\mathbf{u}_{l i b}(x, y)=\nabla \times\left(\Psi \mathbf{e}_{z}\right)$ is the inviscid libration flow computed from Eq. (A3). In Fig. 6, we show the resulting horizontal velocity $u_{x, y}^{\prime}$ and the corresponding vertical vorticity $\omega_{z}^{\prime}(x, y)$ fields for both frequencies, for the same phases $\sigma_{0} t=0$ and $\pi / 2$ as previously. Here again, measurements are not displayed in lateral strips of width $6 \mathrm{~mm}$ along the walls because of the poor quality of the PIV data.

For both frequencies, the horizontal flow shows a complex spatial structure, which verifies the relation $u_{x, y}^{\prime}(-x,-y)=-u_{x, y}^{\prime}(x, y)$, confirming the $s=+$ symmetry of the flow. Both frequencies show a clear square pattern of vorticity extrema, which is the trace of the interferences between the eight propagative inertial wave beams emitted from the eight horizontal edges of the container. Note that since the libration flow has constant vorticity far from the boundaries, one has $\omega_{z}^{\prime}=\omega_{z}+2 \epsilon \Omega_{0} \cos \left(\sigma_{0} t\right)$, so the vorticity patterns computed from the total flow and from its wave component are identical. The amplitude of the wave component in the bulk of the flow is typically a factor 10 below the libration velocity scale $\epsilon \Omega_{0} L$, indicating a weak efficiency of the excitation of inertial waves by libration.

In Figs. 6(c) and 6(f), we show for comparison the numerical predictions of the inviscid modes $[2,1,+]$ and $[2,2,+]$ in the horizontal plane. The mode $[2,1,+]$ (c) consists in a nearly axisymmetric flow, in which each velocity vector describes an elliptic oscillation in the anticyclonic direction. During one period of the mode, the flow goes through the following sequence: cyclonic, centrifugal, anticyclonic, centripetal (see Ref. 32). The mode $[2,2,+]$ (f) has a very different structure: it has four vortices located along the sidewalls, with a hyperbolic point in the middle. This pattern is essentially 
rotating as a whole around the rotation axis, with only weak deformations near the walls due to the non-axisymmetry of the container.

The comparison between the numerical inviscid modes and the experimental measurements is revealing. The structure of the mode $[2,1,+]$ can be easily recognized in the experimental data, superimposed to the square pattern of the interfering edge waves. For instance, the numerical mode chosen at the phase shown in Fig. 6(c) matches well the experimental flow at the libration phase $\sigma_{0} t$ $=0$ [Fig. 6(a)]. At this phase, the flow is normal to the plane $y=0$, in agreement with the vanishing of the mode in the vertical plane observed at the same phase in Fig. 5(a). On the other hand, the structure of the inviscid mode $[2,2,+]$ cannot be recognized in the experimental data at any phase, ${ }^{32}$ confirming that this mode cannot be excited by libration. For both frequencies, the vorticity field is essentially dominated by the interfering edge waves, and is always different from the vorticity of the predicted inviscid modes [Figs. 6(c) and 6(f)].

The absence of mode $[2,2,+]$ in the experimental fields may be understood from symmetry arguments. In addition to the $s=+$ symmetry, which corresponds to an invariance under a rotation of $\pi$ about the $z$ axis, the $[2,1,+]$ mode also turns out to be invariant under a rotation of $\pi / 2$ about the $z$ axis [Fig. 6(c)], which exactly matches the symmetry of the libration forcing for a cube. On the contrary, the mode $[2,2,+]$ has only the $\pi$ symmetry, and it is antisymmetric with respect to the rotation of $\pi / 2$ about $z$. Taking into account this additional symmetry explains why only $[2,1,+]$ is excited by the libration and not $[2,2,+]$, even when the forcing frequency exactly matches the predicted frequency. Excitation of $[2,2,+]$ would require two adjacent walls to move in opposition of phase, which is not possible with a rigid container.

These observations confirm that the libration forcing is able to excite only a subset of the possible inertial modes. Excitable modes $[n, m, s]$ have even $n$ and $s=+$, and must in addition be symmetric under the rotation of $\pi / 2$ about $z$, which is satisfied for $[2,1,+]$ but not for $[2,2,+]$.

\section{RESONANCE CURVE}

We finally describe the energy of the oscillating flow as the libration amplitude $\epsilon$ and the forcing frequency $\sigma_{0} / 2 \Omega_{0}$ are varied. First, we have varied the libration amplitude $\epsilon$ for the two frequencies $\sigma_{0} / 2 \Omega_{0}=0.648$ and 0.674 corresponding to the (excitable) mode $[2,1,+]$ and the (non excitable) mode $[2,2,+]$. The energy of the wave component $\mathbf{u}^{\prime}(x, y, t)$ of the flow [see Eq. (9)] is plotted in Fig. 7 as a function of $\epsilon$ for these two frequencies. This energy is computed over the horizontal components of the velocity in the horizontal plane $z=4 L / 3$,

$$
k_{h}=\overline{\left\langle u_{x}^{\prime 2}+u_{y}^{\prime 2}\right\rangle_{x, y}},
$$

where the overbar stands for time average.

For both frequencies, the scaling of the energy is compatible with $\epsilon^{2}$, confirming that the wave component of the flow essentially responds linearly to the excitation amplitude $\epsilon$ in the range

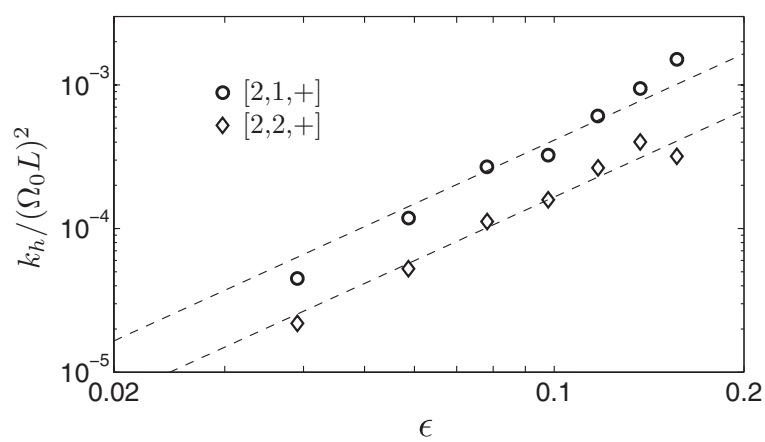

FIG. 7. Normalized kinetic energy $k_{h} /\left(\Omega_{0} L\right)^{2}$ of the wave component of the flow in the horizontal plane $z=4 L / 3$ as a function of the excitation amplitude $\epsilon$, for libration frequencies corresponding to the $[2,1,+](\circ)$ and $[2,2,+](\diamond)$ modes. The dashed lines show the best fits in $\epsilon^{2}$. 


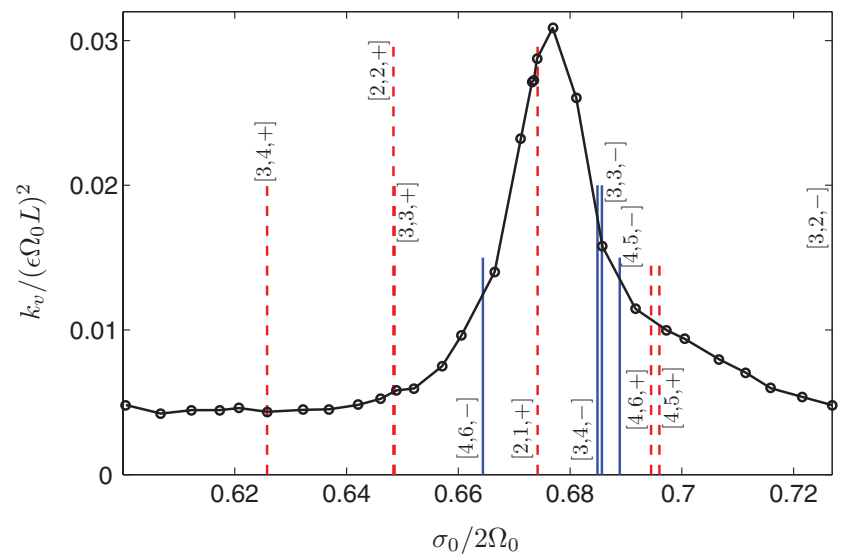

FIG. 8. Normalized kinetic energy $k_{v} /\left(\epsilon \Omega_{0} L\right)^{2}$ in the vertical plane $y=0$ as a function of the excitation frequency $\sigma_{0} / 2 \Omega_{0}$ for an excitation amplitude $\epsilon=0.02$. The vertical lines indicate the eigen frequencies of the inviscid modes of low order (only modes with $n \leq 4$ and $m \leq 6$ are shown). Solid lines correspond to $s=-$ modes and dashed lines to $s=+$ modes. The size of the vertical segments is taken proportional to $1 / n$.

$0.02-0.16$. The energy for the frequency corresponding to $[2,1,+]$ is a factor 2.5 above that for $[2,2,+]$. Since we know that the mode $[2,2,+]$ is not excited, the curve for $[2,2,+]$ essentially corresponds to the interfering propagating edge beams. The additional amount of energy in $[2,1,+]$ therefore corresponds to the resonant mode $[2,1,+]$. These curves suggest that, in the horizontal plane, the energy of the mode $[2,1,+]$ and that of the edge waves are of the same order.

In order to characterize in more detail the resonance of the mode $[2,1,+]$, we have also computed the energy in the vertical plane $y=0$,

$$
k_{v}={\overline{\left\langle u_{x}^{2}+u_{z}^{2}\right\rangle}}_{x, z}
$$

The libration flow being normal to the measurement plane, it does not contribute to $k_{v}$, so this definition essentially describes the energy of the wave component of the flow. Focusing on a single value of the excitation amplitude, $\epsilon=0.02$, we have performed a systematic scan over the excitation frequency $\sigma_{0} / 2 \Omega_{0}$ in the range [0.60; 0.73]. Since the energy basically scales as $\epsilon^{2}$, the energy $k_{v}$ in Fig. 8 is normalized now by $\left(\epsilon \Omega_{0} L\right)^{2}$, which represents the order of magnitude of the energy of the basic libration flow.

The resonance curve shows a well-defined peak of energy $k_{v} \simeq 3 \times 10^{-2}\left(\epsilon \Omega_{0} L\right)^{2}$, centered on $\sigma_{0} / 2 \Omega_{0}=0.676 \pm 0.003$, and of width at half maximum $\Delta \sigma / 2 \Omega_{0} \simeq 0.02$. The peak frequency is very close to the numerical prediction for the inviscid mode $[2,1,+], \sigma_{0} / 2 \Omega_{0}=0.6742$, confirming that this mode is the only one which is significantly excited in the frequency range investigated here. We can note a slight shift of about $\sigma_{0} / 2 \Omega_{0} \simeq+0.002$ between the predicted and the observed peak, which probably originates from a viscous detuning effect. Although this detuning has not been computed for the inertial modes of a parallelepiped, it is essentially similar to the one found for the inertial modes of a sphere. ${ }^{1}$ The frequency shift for the sphere has been actually determined analytically, and is of the order of $\sigma_{0} / 2 \Omega_{0} \simeq(0.02-0.05) \sqrt{E}$ for low order modes. This would give $\sigma_{0} / 2 \Omega_{0} \simeq 0.002-0.004$ for $E=5.3 \times 10^{-5}$, which is consistent with the shift observed here for the cube.

The resonance peak is surrounded by a nearly constant plateau at $k_{v} \simeq(5 \pm 1) \times 10^{-3}\left(\epsilon \Omega_{0} L\right)^{2}$, which accounts for the wave beams emitted from the edges of the container. This plateau confirms that the edge beams are always present in the flow, and are hidden only at the particular frequencies at which a mode is excited. It is worth noting that the width of the resonance peak is significantly larger than the width of the peak at $\sigma_{0}$ in the temporal energy spectra of the flow (see Fig. 3). This suggests that the quality of the resonance of the mode $[2,1,+]$ is not fixed by the precision of the libration forcing, but is rather governed by the viscosity. This is confirmed by the fact that the 
resonance width $\Delta \sigma / 2 \Omega_{0} \sim 0.02$ is of the order of $\left(\Omega_{0} \tau_{E}\right)^{-1} \simeq 0.01$, where $\tau_{E}=L / \sqrt{\nu \Omega_{0}}$ is the Ekman time scale.

As expected, inertial modes which do not have the symmetries of the libration forcing (e.g., $[3,4,+]$ or $[3,3,+]$, with odd $n)$ are not found in the resonance curve. More interestingly, the even and symmetric (with respect to rotation of $\pi$ ) mode $[2,2,+]$ is not found either, confirming that the antisymmetry (with respect to rotation of $\pi / 2$ ) of this particular mode is not compatible with the symmetry of the libration. However, it appears that at this frequency, the system still lies in the far tail of the $[2,1,+]$ resonance, which probably explains the residual flow structure associated to the mode $[2,1,+]$ found in Fig. 5(e). We can conclude that, because of viscous effects and symmetry properties, the libration forcing selects only one single resonant inertial mode in the range [0.60; 0.73]. The slight asymmetry in the tails of the resonance curve might be due to the presence of the modes $[4,5,+]$ and $[4,6,+]$ at frequencies slightly larger than the $[2,1,+]$ peak. It turns out that $[4,5,+]$ has the $\pi / 2$ symmetry, whereas $[4,6,+]$ has the $\pi$ symmetry only; it is therefore conceivable that a slight contribution of the mode $[4,5,+]$ is responsible for the asymmetric shape of the resonance curve.

\section{EXCITATION OF INERTIAL MODES BY THE EDGE BEAMS}

The present results raise the question of the relation between the inertial modes and the edge beams originating from the convergence of Ekman fluxes near the edges of the container. Interestingly, for forcing frequencies close to the frequency of low-order symmetric inertial modes $[n, 1,+]$ with even $n$, the edge beams form a periodic ray pattern in the vertical plane $(x, z)$. This is illustrated in Figs. 9(a) and 9(c) for libration frequencies corresponding to the modes $[2,1,+]$ and $[4,1,+]$, showing one and two X-shaped patterns, respectively. This figure suggests that the periodicity of the rays leads to the formation of a standing wave, since energy can propagate along these rays either way. It motivates the use of a two-dimensional simplification, in which the planar wave beams emitted from the edges of the cube propagate in the vertical plane along rays, at an angle given by the dispersion relation (7). This 2D approximation does not apply near the corners, where the flow must be three-dimensional. But away from the walls, the edge beams essentially protrude in the bulk of the fluid, as if the container were infinitely long in the along-edge direction.

We consider, in the following, the 2D ray orbits in a vertical plane originating from the edges of the cube. These orbits can be characterized by integers, $(i, j)$, denoting the number of reflections from vertical $(i)$ and horizontal $(j)$ boundaries. Here, we count edge reflections as reflections on both the vertical as well as the horizontal walls. The orbits in Figs. 9(b) and 9(d) therefore classify as $(1,1)$ and $(2,1)$ periodic orbits, respectively. The angle which the beam makes with the horizontal is $\theta_{i j}=\tan ^{-1}(j / i)$, yielding a normalized frequency

$$
\frac{\sigma_{i j}}{2 \Omega_{0}}=\cos \left(\tan ^{-1}\left(\frac{j}{i}\right)\right)=\frac{i}{\sqrt{i^{2}+j^{2}}},
$$

similar to the expression for the eigenfrequencies of the transverse modes of an infinite channel. ${ }^{9}$ The frequencies $\sigma_{i j}$ are dense in $\left[0,2 \Omega_{0}\right]$. This spectrum is degenerate: patterns $(i, j)$ and $\left(i^{\prime}, j^{\prime}\right)$ have the same frequency if $i^{\prime} / i=j^{\prime} / j$ (such degeneracy is not obvious for the inertial modes in the parallelepiped). The resulting ray pattern may be associated with a set of cells, with hyperbolic points at the intersection between rays. Restricting to ray patterns compatible with symmetries of the libration, this construction yields $2 i \times 2 j$ cells [see dashed lines in Figs. 9(b) and 9(d)], corresponding to an $n=2 i$ inertial mode structure. No similar connection can be made between index $m$ and $j$ from this simple geometrical construction, because $m$ is not related to the number of cells in the horizontal direction.

If we compare the frequencies of the periodic patterns $(i, j)$ and the frequencies of the inertial modes $[n, m, s]$, we obtain a reasonable agreement for $n=2 i, m=1$, and $s=+$ (see Table II). The discrepancy is $4.7 \%$ for $[2,1,+]$, and decreases for larger $n$. We can also note that, for $j>1$, it is always possible to find, among the modes having $n=2 i$ and $s=+$, a mode $m>1$ such that $\sigma_{i j} \simeq \sigma_{n m s}$ to within a few $\%$. For instance, for $(i, j)=(1,2)$, one has $\sigma_{12} / 2 \Omega_{0}=0.447$, which is close to the frequencies of modes $[2,4,+]$ and $[2,5,+]$ (0.465 and 0.434 , respectively). One 

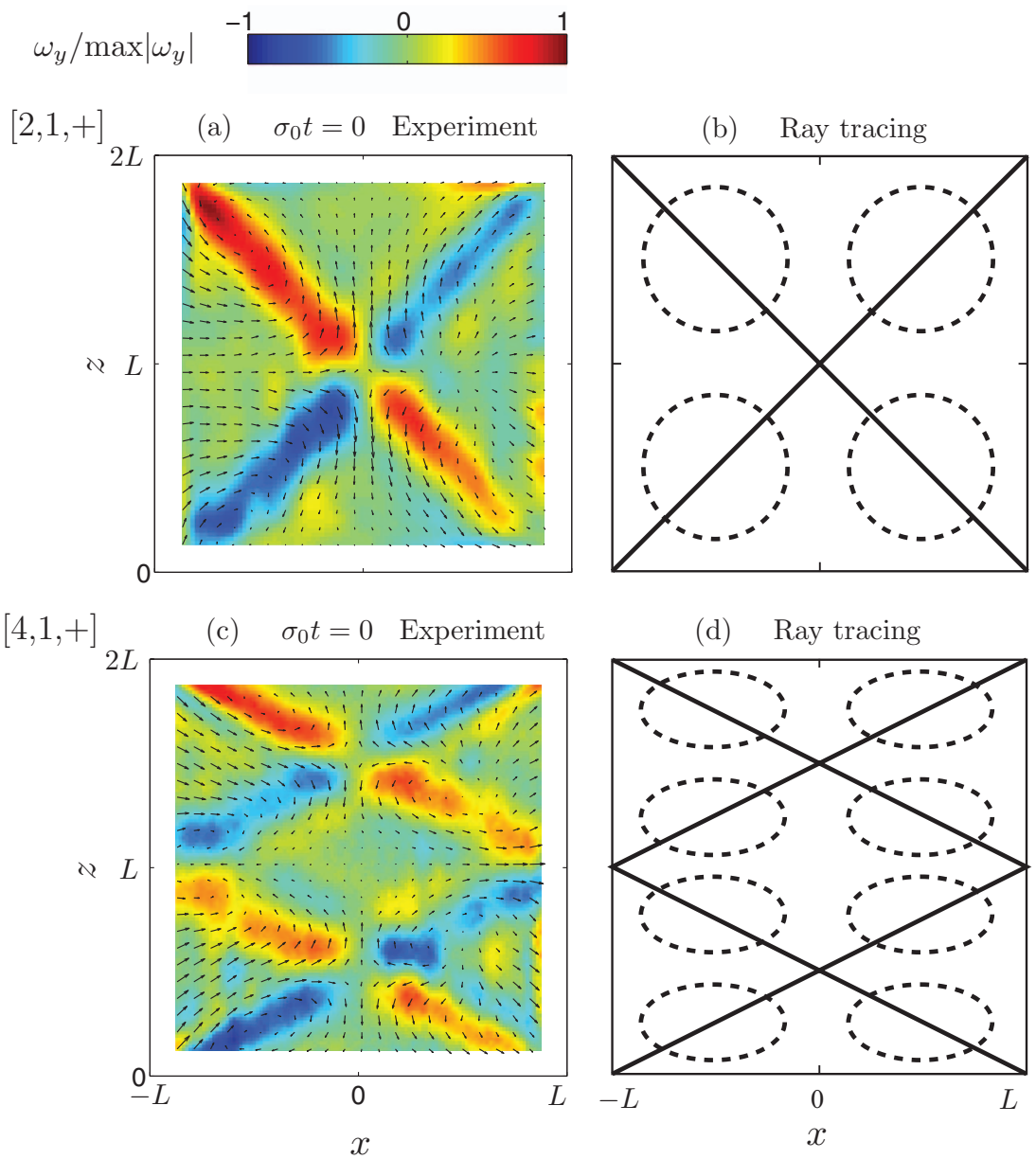

(d) Ray tracing

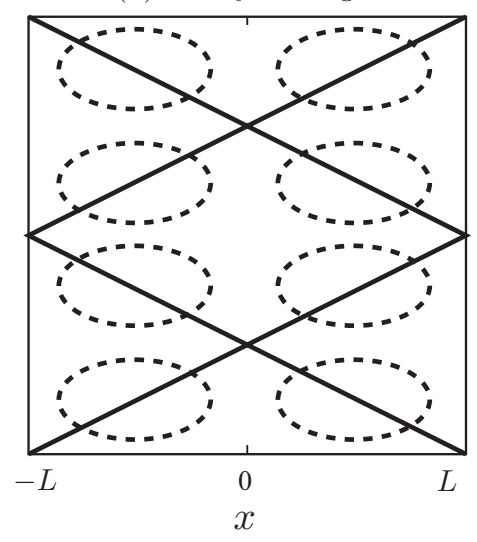

FIG. 9. (a) and (c) Flow in the vertical plane $y=0$ corresponding to the modes $[2,1,+]$ and $[4,1,+]$; (b) and (d) corresponding ray-tracing patterns $(1,1)$ and $(2,1)$. Figures are taken at the libration phase $\sigma_{0} t=0$, for which, the mode has zero amplitude, so only the wave beams are visible.

explanation for the apparent ability of strictly $2 \mathrm{D}$ periodic orbits to predict eigenfrequencies of the 3D problem quite accurately may lie in the fact that while the eigenspectrum is dense, there are indications that the density of states (the amount of eigenfrequencies per frequency increment) may be nonuniform. Computations in long channels ${ }^{9}$ show that eigenfrequencies cluster preferentially around the eigenfrequencies of strictly transverse modes of an infinitely long channel, and there are indications that this also applies to the higher vertical modes (large $n$ ) in a cube.

The coincidence between the frequencies of inertial modes and periodic beam patterns of similar spatial structure suggests the following mechanism for the excitation of modes in a librated container. A given libration frequency induces a set of inertial wave beams originating from the detachment of the oscillating Ekman layers at the edges. If the libration frequency is close to an inertial mode,

TABLE II. Comparison between the frequencies of the periodic edge beam patterns $(i, 1)$ and the frequencies of the inertial modes with $[n, 1,+]=[2 i, 1,+]$.

\begin{tabular}{lccccc}
\hline \hline Pattern $(i, j)$ & $\theta_{i j}$ & $\sigma_{i j} / 2 \Omega_{0}$ & Mode $[n, m, s]$ & $\sigma_{n m s} / 2 \Omega_{0}$ & Difference (\%) \\
\hline$(1,1)$ & $45^{\circ}$ & 0.707 & {$[2,1,+]$} & 0.674 & 4.7 \\
$(2,1)$ & $26.6^{\circ}$ & 0.894 & {$[4,1,+]$} & 0.875 & 2.1 \\
$(3,1)$ & $18.4^{\circ}$ & 0.949 & {$[6,1,+]$} & 0.938 & 1.2 \\
\hline \hline
\end{tabular}


the resulting edge beam pattern is nearly periodic, and has the same symmetries as the mode-this is at least the case for the two modes $[2,1,+]$ and $[4,1,+]$ shown in Fig. 9. This suggests that the edge beams can supply energy to the modes directly in the bulk of the flow, with the correct vertical periodicity. The libration flow, being associated to $n=0$ (vertical invariance), is unable to excite these modes other than viscously, through the edge beams, because it lacks the vertical structure of the inertial modes. This may be different in the presence of sloping boundaries, which enforce vertical motions, and may excite inertial waves inviscidly. We may conclude that inertial modes are excited with an inertial velocity scale $\epsilon \Omega_{0} L$, but this excitation requires the presence of edge beams induced by the viscous oscillating Ekman layers.

\section{CONCLUSION}

In symmetric containers, i.e., axisymmetric containers or containers whose side walls are either parallel or perpendicular to the rotation axis, longitudinal libration produces an inviscid type of fluid response that conserves absolute vorticity. However, by friction at bottom, top, and side walls, this leads to a periodic mass flux in boundary layers adjacent to the horizontal walls. Convergence and divergence of these Ekman fluxes near the edges spawn free shear layers that are inclined with respect to the rotation axis. ${ }^{16,25,33}$ Their angle is set by the ratio of the modulation frequency to the Coriolis frequency. Expulsion of Ekman mass fluxes takes place where the topography has the same inclination, at so-called critical slopes. In containers where changes in the orientation of the boundary occur suddenly (such as near the edges of the cube studied here), these critically sloping regions are "buried" in these horizontal edges. The free shear layers thus spawn from the edges, and are here termed "edge beams."

In a $2 \mathrm{D}$ approximation, these edge beams propagate along periodic paths. For paths that are short enough, upon a few reflections energy folds back onto itself and an inertial mode may establish itself: a vertically standing large-scale structure that may either be propagating, or standing ("sloshing") horizontally. In our experiment, symmetries, due to the way that the fluid is forced into motion by means of libration, allow the realization of only a particular subset of all possible theoretical inertial modes. Latitudinal libration (libration along an axis normal to the rotation axis) might provide a mechanism by means of which also the other, anti-symmetric modes can be excited, e.g., the mode $[1,1,-]$, which consists in a single oscillating cell.

Previous studies in cylindrical domains suggest that the resonant forcing (and, therefore, amplification) of a vertically standing inertial mode opens the possibility of elliptic instability (vortex breakdown). This should be manifested as a sudden collapse of the inertial wave mode. This has so far not been seen in our experiments in a cube, but is not ruled out either. Another peculiarity of the theoretical inviscid wave field in the cube is the appearance of many horizontal scales of motion, even for "large-scale" modes (i.e., having low vertical wave number and high frequency). ${ }^{9}$ These scales are required by the wave field in order to match the rotational (circular) to the geometrical (square) symmetries. A more detailed experimental observation of an inertial mode is required to investigate this property. Finally, it is of interest to examine as to what extent edge beams and inertial modes can play a role in containers whose symmetry is broken.

\section{ACKNOWLEDGMENTS}

We acknowledge A. Aubertin, L. Auffray, C. Borget, and R. Pidoux for experimental help, and Y. Duguet, W. Herreman, and M. Rabaud for fruitful discussions. J.B. is supported by the "Triangle de la Physique." This work is supported by the ANR (Grant No. ANR-2011-BS04-006-01) "ONLITUR." The rotating platform "Gyroflow" was funded by the "Triangle de la Physique."

\section{APPENDIX: THE INVISCID BASE FLOW IN A LIBRATING CUBE}

In this section, we derive the streamfunction describing the inviscid flow generated by the libration of a parallelepiped container of square cross section. The Euler equation in the librated 
frame of reference writes

$$
\frac{\partial \mathbf{u}}{\partial t}+(\omega+2 \Omega) \times \mathbf{u}=-\nabla\left(\frac{p}{\rho}+\frac{1}{2}|\Omega(t) \times \mathbf{r}|^{2}+\frac{1}{2}|\mathbf{u}|^{2}\right)-\frac{d \Omega}{d t} \times \mathbf{r},
$$

with $\boldsymbol{\Omega}(t)$, the total angular velocity, of vertical component given by Eq. (1). This equation contains three terms related to the (modulated) background rotation: (1) the Coriolis acceleration (last term on left-hand side), (2) the centrifugal force (second term on right-hand, that can be absorbed in a reduced pressure), and (3) the Euler force (last term on right-hand side), due to the modulation of the rotation rate. ${ }^{34}$ Here, $\omega$ is the relative vorticity vector, $\omega=\nabla \times \mathbf{u}$. Taking the curl of the Euler equation (A1), we obtain the vorticity equation:

$$
\frac{\partial \boldsymbol{\omega}}{\partial t}+\mathbf{u} \cdot \nabla(\boldsymbol{\omega}+2 \Omega)-(\boldsymbol{\omega}+2 \Omega) \cdot \nabla \mathbf{u}=-2 \frac{d \boldsymbol{\Omega}}{d t} .
$$

This equation tells that relative vorticity changes due to advection of absolute vorticity,

$$
\omega_{a}=\omega+2 \Omega
$$

(second term on left-hand side), vortex tilting by sheared motion (third term), or by changes in the rotation rate (right-hand side).

Note that for forcing by libration, the driving term on the right of the vorticity equation is present in the vertical direction only and is independent of the vertical coordinate. Since the boundaries are not inclined relative to the rotation axis, this motivates looking for velocity fields that are $z$-independent as well. In fact, the vanishing of the vertical velocity at the horizontal bottom and top walls suggests looking for solutions of zero vertical velocity. The vertical component of the vorticity $\omega_{a}=\omega_{a} \cdot \mathbf{e}_{z}$ (which at this point is the only nonvanishing component) thus satisfies

$$
\frac{\partial \omega_{a}}{\partial t}=0
$$

Since the horizontal velocity field is nondivergent $\left(\partial_{x} u_{x}+\partial_{y} u_{y}=0\right)$, we can introduce a streamfunction such that $\left(u_{x}, u_{y}\right)=\left(-\partial_{y} \psi, \partial_{x} \psi\right)$. The spatial part $\Psi(x, y)$ of streamfunction, $\psi(x, y, t)$ $=\Psi(x, y) \cos \left(\sigma_{0} t\right)$, therefore satisfies a Poisson equation,

$$
\Delta \Psi=-2 \epsilon \Omega_{0},
$$

subject to the condition that in the librating frame, the solid boundary at $x= \pm L$ and $y= \pm L$ acts as a streamline. We introduce the normalized coordinates $(\tilde{x}, \tilde{y})=(x, y) / L$. The solution of Eq. (A2) is the sum of a particular solution, $\Psi_{p}$, and a homogeneous solution, $\Psi_{h}$, such that $\Delta \Psi_{h}=0$. A particular solution is

$$
\Psi_{p}=\epsilon \Omega_{0}\left(1-\tilde{x}^{2}\right),
$$

that matches the right hand forcing term and satisfies the boundary condition at $\tilde{x}= \pm 1$, but is nonvanishing at $\tilde{y}= \pm 1$. The homogeneous solution, $\Psi_{h}$, also vanishes at $\tilde{x}= \pm 1$, but annihilates $\Psi_{p}(\tilde{x})$ at $\tilde{y}= \pm 1$. It is given by

$$
\Psi_{h}=-4 \epsilon \Omega_{0} \sum_{n=0}^{\infty}(-1)^{n} \frac{\cos \left(d_{n} \tilde{x}\right) \cosh \left(d_{n} \tilde{y}\right)}{d_{n}^{3} \cosh \left(d_{n}\right)},
$$

where

$$
d_{n} \equiv(2 n+1) \frac{\pi}{2}
$$

For convenience, we finally symmetrize the solution, by simply computing $(\Psi(\tilde{x}, \tilde{y})+\Psi(\tilde{y}, \tilde{x})) / 2$, yielding

$$
\Psi=\epsilon \Omega_{0}\left(1-\frac{1}{2}\left(\tilde{x}^{2}+\tilde{y}^{2}\right)-2 \sum_{n=0}^{\infty}(-1)^{n} \frac{\cos \left(d_{n} \tilde{x}\right) \cosh \left(d_{n} \tilde{y}\right)+\cos \left(d_{n} \tilde{y}\right) \cosh \left(d_{n} \tilde{x}\right)}{d_{n}^{3} \cosh \left(d_{n}\right)}\right) .
$$


${ }^{1}$ H. Greenspan, The Theory of Rotating Fluids (Cambridge University Press, London, 1968).

2 J. Pedlosky, Geophysical Fluid Dynamics (Springer-Verlag, Heidelberg, 1987).

${ }^{3}$ J. Lighthill, Waves in Fluids (Cambridge University Press, London, 1978).

${ }^{4}$ A. D. McEwan, "Inertial oscillations in a rotating fluid cylinder," J. Fluid Mech. 40, 603-639 (1970).

${ }^{5}$ K. K. Zhang, X. H. Liao, and P. Earnshaw, "On inertial waves and oscillations in a rapidly rotating spheroid," J. Fluid Mech. 504, 1-40 (2004).

${ }^{6}$ O. M. Phillips, "Energy transfer in rotating fluids by reflection of inertial waves," Phys. Fluids 6(4), 513 (1963).

${ }^{7}$ A. A. M. Manders and L. R. M. Maas, "Observations of inertial waves in a rectangular basin with one sloping boundary," J. Fluid Mech. 493, 59 (2003).

${ }^{8}$ G. K. Batchelor, An Introduction to Fluid Dynamics (Cambridge University Press, Cambridge, 1967)

${ }^{9}$ L. R. M. Maas, "On the amphidromic structure of inertial waves in rectangular parallelepiped," Fluid Dyn. Res. 33, 373-401 (2003).

${ }^{10}$ K. D. Aldridge and A. Toomre, "Axisymmetric inertial oscillations of a fluid in a rotating spherical container," J. Fluid Mech. 37, 307-323 (1969).

${ }^{11}$ M. Rieutord, "Linear theory of rotating fluids using spherical harmonics. II. Time-periodic flows," Geophys. Astrophys. Fluid Dyn. 59, 185-208 (1991).

12 A. Tilgner, "Driven inertial oscillations in spherical shells," Phys. Rev. E 59, 1789-1794 (1999).

${ }^{13}$ J. Noir, F. Hemmerlin, J. Wicht, S. Baca, and J. Aurnou, "An experimental and numerical study of librationally driven flow in planetary cores and subsurface oceans," Phys. Earth Planet Inter. 173, 141-152 (2009).

${ }^{14}$ M. A. Calkins, J. Noir, J. D. Eldredge, and J. M. Aurnou, "Axisymmetric simulations of libration-driven fluid dynamics in a spherical shell geometry," Phys. Fluids 22, 086602 (2010).

${ }^{15}$ F. H. Busse, "Steady fluid flow in a precessing spheroidal shell," J. Fluid Mech. 33(4), 739 (1968).

${ }^{16}$ R. R. Kerswell, "On the internal shear layers spawned by the critical regions in oscillatoray Ekman boundary layers," J. Fluid Mech. 298, 311 (1995).

${ }^{17}$ J. Noir, D. Jault, and P. Cardin, "Numerical study of the motions within a slowly precessing sphere at low Ekman number," J. Fluid Mech. 437, 283 (2001).

${ }^{18}$ S. T. Suess, "Viscous flow in a deformable rotating container," J. Fluid Mech. 45, 189-201 (1971).

${ }^{19}$ C. Morize, M. Le Bars, P. Le Gal, and A. Tilgner, "Experimental determination of zonal winds driven by tides," Phys. Rev. Lett. 104, 214501 (2010).

${ }^{20}$ W. V. R. Malkus, "Precession of earth as cause of geomagnetism," Science 160, 259 (1968).

${ }^{21}$ A. Tilgner, "Zonal wind driven by inertial modes," Phys. Rev. Lett. 99, 194501 (2007).

${ }^{22}$ S. B. Dalziel, "Decay of rotating turbulence: Some particle tracking experiments," Appl. Sci. Res. 49, 217-244 (1992).

${ }^{23}$ G. P. Bewley, D. P. Lathrop, L. R. M. Maas, and K. R. Sreenivasan, "Inertial waves in rotating grid turbulence," Phys. Fluids 19, 071701 (2007).

${ }^{24}$ C. Lamriben, P.-P. Cortet, F. Moisy, and L. R. M. Maas, "Excitation of inertial modes in a closed grid turbulence experiment under rotation," Phys. Fluids 23, 015102 (2011).

${ }^{25}$ Y. Duguet, J. F. Scott, and L. Le Penven, "Oscillatory jets and instabilities in a rotating cylinder," Phys. Fluids 18, 104104 (2006).

${ }^{26}$ DaVis, LaVision GmbH, Anna-Vandenhoeck-Ring 19, Goettingen, Germany.

${ }^{27}$ F. Moisy, PIVMat toolbox for Matlab, Laboratory FAST, University Paris-Sud, France.

${ }^{28}$ A. Sauret, D. Cébron, M. Le Bars, and S. Le Dizès, "Fluid flows in a librating cylinder," Phys. Fluids 24, 026603 (2012).

${ }^{29}$ K. S. Peat, "Internal and inertial waves in a viscous rotating stratified fluid," Appl. Sci. Res. 33(5-6), 481-499 (1977).

${ }^{30}$ A. Tilgner, "Oscillatory shear layers in source driven flows in an unbounded rotating fluid," Phys. Fluids 12, 1101 (2000).

${ }^{31}$ P.-P. Cortet, C. Lamriben, and F. Moisy, "Viscous spreading of an inertial wave beam in a rotating fluid," Phys. Fluids 22, 086603 (2010).

${ }^{32}$ See supplementary material at http://dx.doi.org/10.1063/1.4731802 for movies of the experimental fields compared to the numerical inviscid modes.

${ }^{33}$ A. Swart, L. R. M. Maas, U. Harlander, and A. Manders, "Experimental observation of strong mixing due to internal wave focusing over sloping terrain," Dyn. Atmosph. Oceans 50, 16-34 (2010).

${ }^{34}$ I. Tolstoy, Wave Propagation (McGraw-Hill, New York, 1973). 